\title{
Modelling for Clinical and Psysiological Evaluation of Diabetes and Glucose Homeostasis
}

\author{
Elirea Bornmann \\ ${ }^{1}$ Faculty of Pharmacy, Rhodes University, Makhanda, 6139, South Africa. \\ 1elirea885@outlook.com
}

\author{
Article Info \\ Journal of Biomedical and Sustainable Healthcare Applications (http://anapub.co.ke/journals/jbsha/jbsha.html) \\ Doi: https://doi.org/10.53759/0088/JBSHA202202004 \\ Received 20 January 2021; Revised form 18 March 2021; Accepted 25 July 2021. \\ Available online 05 January 2022. \\ (C)2022 Published by AnaPub Publications.
}

\begin{abstract}
There is a long history of arithmetic simulations in the domain of gluconeogenesis. There are various reasons why frameworks are used. Paradigms have been employed to calculate physiologically relevant parameters from intermediate experimental evidence, to offer a clear quantitative description of pathophysiology processes, and to identify clinical relevance indicators from basic empirical procedures. The creation and application of frameworks in this field has expanded in response to the rising social effect of type 2 diabetes that entails a disruption of the glycemic homeostasis system. The frameworks' emphasis has ranged from depictions of entire body functions to lymphocytes (form "in Vivo" to "in Vitro") study, following the methodologies of physiologic and medicinal exploration. Framework-based techniques to connecting in vivo and in vitro research, and also multi-resolution systems that combine the two domains, have been presented. The arithmetic and psychological domains have had varying levels of effectiveness and influence.
\end{abstract}

Keywords - Physiological Modeling Functions, Glycemic Metabolism, Glycemic Homeostasis, Thyroxine Production

\section{INTRODUCTION}

Designs that are widely used in medical implementations, such as for assessing glycemic tolerance and cell function, and modeling techniques that symbolize particular elements of the glycemic homeostasis framework, that have been iconic for their effectiveness in defining key physiological modelling functions, such as thyroxine production from pancreatic islets, have piqued interest. Designs of a physical system are always reduced and imprecise depictions. A suitable mix of faithfulness to truth, comprehensibility, interpretive value, and practical utility is critical to their success. This has been accomplished using a number of methods. Despite the fact that several simulations for the glycemic homeostasis mechanism have been suggested, study in this field still needs to tackle a number of challenges and explore new avenues. The quantitative depiction of glycemic homeostasis mechanisms is somewhat incomplete, partly due to the fact that certain processes are yet unknown. Statistical methods have yet to reach their full potential in in vitro study. This study focuses on the most important and engaging frameworks to show the difficulties, techniques, and contributions of arithmetic analysis to the physiology and clinical exploration of glycemic metabolism and diabetes.

In the study of glycemic metabolism, computational analysis has a long history. In the early 1960s, one of the first "minimal" frameworks of the glycemic-thyroxine network was presented. Glycemic metabolism has previously been extensively researched utilizing glycemic projectiles and statistical frameworks. Tham et al in [1] laid the groundwork for glycemic sampling simulation, characterizing a three-compartment framework for branded glycemic kinetics and a singlecompartment design for glycemic manufacturing evaluation in non-stationary circumstances, which has since become a requirement and is still in use. Arithmetic frameworks for metabolic tracers blossomed in the previous years, for example, in the Bulleted lists of Arithmetic Biophysics, which was created in 1939. Modeling of thyroxine secretion is a relatively new field; given the thyroxine radioimmunoassay has only been accessible since the early 1960s. Chewning, Baker, Pipkin, Douglas, and Mitchell in [2] provided a key framework of the complicated thyroxine secretion patterns seen in numerous "in Vivo" to "in Vitro" assessments in the early cell study in the 70s. The application of statistical models has been presented into the major line of metabolism study from early years. The increasing globalized concerns on the Type 2 Diabetes (T2D), a significant condition of glycemic homeostasis, have sparked more metabolism study, especially framework-based investigations.

Through many instances, we present the principles, issues, techniques, goals, and successes in the field of glycemic homeostasis in background analyses with major focus on psychological modeling in this article. A full assessment is impossible due to the enormous variety of systems in this field, which range from biochemical processes in cells through clinical testing simulation. Jayasekera et al in [3] provide a general overview, discussing a wide range of frameworks with various implementations. We've chosen to focus on a few frameworks that we believe are important in the physiology and clinical research of glycemic metabolism and T2D. As this multidisciplinary topic necessitates, our target readership comprises researchers from both the arithmetic and psychological domains. We think that by doing this evaluation, we will 
be able to strengthen the mutual interest and trust between these two categories. With that regard, this paper has been organized as follows: Section II focuses on arithmetic modeling background. Section III critically evaluates the psychological modeling aspect. Section IV draws conclusions to the article.

\section{ARITHMETIC MODELING BACKGROUND}

Ideas and Aims

The organic idea behind using statistical frameworks in metabolism, as well as other specialists, is to apply the effective science framework to comprehend, evaluate, and estimate physiological modelling functions. This methodology has some implementations in the research of metabolic activities in its company regulations. The arithmetical concepts of tracers have shown that rigorous approaches may be employed to illustrate the methodologies' important. This is an anomaly, since most systems have considerable approximations that make their utility and success more challenging.

In physical study, arithmetic formulation has been utilized for a number of goals. A modest but essential aim has been the assessment of elements that are not immediately measured. The so-called Fick's approach is founded on simple calculations for conservation of momentum and convection transportation of a material in a fluid for predicting cardiac output. A comparable necessity spurred the creation of tracer techniques, e.g. the Steele framework or the prevailing standard methodology for evaluating thyroxine sections in vivo. This type of frameworks, like Fick's principle [4], may incorporate hypotheses that are either highly strong or not always suitable, as in Steele's framework. As a consequence, frameworks for this function have gradually evolved, such as Steele's framework.

This group of designs is broadened to include frequently used "computers" of physiological factors, which cannot be evaluated directly or require sophisticated researches. A comprehensive illustration of a fundamental and accurate description of the glycemic-thyroxine homeostasis system intended at assessing thyroxine sensitivity, or thyroxine's capacity to drive glycemic metabolism, is the "minimum paradigm". Another well-known illustration is the "HOMA" indicators of thyroxine sensitivity and cell functions that were previously formulate from the framework of glycemic homeostasis. The relevancy and productivity of the frameworks have been a major basis of efficacy.

It is a bold strategy to employ simulations as clear quantitative approximations of certain physiological modelling functions with the purpose of comprehending the mechanisms underlying the results. Nagaraj and Kp in [5] thyroxine secretion paradigm is an outstanding sample of how a coherent arithmetic description properly illustrated a series of more complex experimental proof whereas also providing hypotheses on the probable techniques underlying cell response. Another significant sample is Zhu et al in [6] framework, which provided an interesting quantitative formulation of the idea that T2D-related cell mass loss might be due to hyperglycemia-induced cell injury. This sort of framework has a lot of potential since it can provide more precise representation of complex physiological function, but there is numerous opportunities for speculation.

The goal of programming in this field is to offer full depictions of the glycemic homeostasis mechanism, and a variety of approximations of various complexities have been proposed, as shown below. Systems of glycemic metabolism, on the other hand, have the issue of dealing with a massively complex system with unknown numerical characteristics. As a consequence, the simulators' reliability has been questioned. Glycemic homeostasis simulations, on the other hand, have been found to be beneficial in evaluating techniques for the best thyroxine administration regimens in type 1 diabetes, employing glycemic level testing or a synthetic pancreatic. The Archimedes framework was a more comprehensive paradigm that went beyond glycemic metabolism to forecast problems and pharmacoeconomics, and it has lately gotten a lot more interest.

One such sector is the use of designs to help in the finding of drugs to treat T2D. The Food and Drug Administration, for example, has supported this technique, known as Pharmaco-Kinetic/Pharmaco-Dynamic (PKPD) frameworks or pharmacometrics, which is largely geared at delivering sophisticated data processing in the drug development procedure. As a result, the traditional PKPD strategy is directed toward framework-based interpretations of experimental observations (i.e., it ignores frameworks for pure simulation) and relies on population techniques to determine framework parameters. PKPD modeling techniques have been used in the research of glycemic metabolism for goals that aren't necessarily related to drug screening. Frameworks are typically segmented, and well-known subframeworks are regularly used. While evaluating the framework's ability to appropriately represent the data is a standard analytical step, the quantitative representation's conformance to physical understanding receives less emphasis.

\section{In Vitro and in Vivo Modeling}

Systems have been constructed to describe in both vivo and in vitro experimental results since the early applications cited in the Preface. In vivo experiments of glycemic homeostasis and thyroxine secretion are likely to have accounted for the majority of the research. In vitro frameworks, notably for thyroxine production, have garnered a lot of attention lately, because to huge advances in in vitro study, as detailed below. Significantly, modeling allows in vitro and in vivo scientific results to be interpreted with unification processes whenever a similar system with more suitable metric scaling, effectively forecasts both the "in Vivo" to "in Vitro" data. Since "in Vitro" study techniques are critical for understanding cellular processes but cannot be used to evaluate the significance of discoveries in vivo, computational analysis provides a means to bridge the gap between the two. As explained below, this method has been applied for thyroxine secretion. 


\section{Merits and Demerits of Arithmetic Representations}

Most alternative logics have been used to translate physiological systems into arithmetic descriptions. The relationship between physiological and arithmetic, in instance, has varying degrees of tidiness. Some methods try to reduce physiologic complexities into modeling components that captivate the essential physiological properties, with a clear correlation between the quantitative expression (e.g., system variables) and the physiology. One illustration is the referenced thyroxine secretion paradigm, in which a distinctive theory on the underlying mechanism and a straightforward arithmetic expression were established to precisely reflect the dependency of first-phase thyroxine secretion on sugar levels. Other techniques are less interested with the exact physiological relevance of the equations and instead concentrate on the designer's capacity to effectively reflect the data. This mindset is often associated with the usage of compartmental frameworks, which may be quickly created and extended to provide enough versatility to explain data. While this method is not inherently wrong, it does run the danger of producing frameworks that have a muddled or distorted connection with the physical system, as detailed more below. When quantitative depictions are backed up by a formal examination of their properties, which helps to explain the function of hypotheses, they are enhanced. For various frameworks, this has been done on occasions. A formal study of the minimum paradigm has offered answers for some challenges observed throughout its many uses.

\section{Merits and Demerits}

Modeling success and failure may be subjective and fruitless to discuss, since non-scientific factors often impact these results. Nonetheless, certain observations may be helpful in improving mutual knowledge between the analytical and experimental groups.

The algorithms that have an obvious utility for diverse parts of data processing are likely to be the most effective. The HOMA framework, which offers a competed index that can be computed with ease of the thyroxime resistance, has retrieved more than 21000 references; the negligible approach, which has been used for thyroxine susceptibility analysis in many investigations, has earned over 1800 reviews. Different data evaluation methods, e.g. approaches for evaluating thyroxine secretion from C-peptide and the methods for evaluating thyroxine sensitivities and the cell activities from the oral glycemic endurance test, garnered a smaller but still significant number of responses. Frameworks that give meaningful assistance for experiment data analysis are highly welcomed, according to these observations.

The achievement of configurations with impressive arithmetical complexity that are not focused toward potential implementation in investigational data processing, on the other hand, is of specific importance: it highlights the capabilities of numerical techniques to provide intriguing perspectives of supernatural events that may be fascinating even if they do not adhere to the experimental observations. Simulations of thyroxine secretion and cell failure in T2D are two illustrations. On the other hand, some systems are unquestionably an experiment with a poor justification. Frameworks that have a poor link with experimental results and include problematic hypotheses should be avoided. Modeling studies, on the other hand, are often neglected owing to the difficulty of expressing the core conclusions to a larger audience, which is inherent in the arithmetic notation. Making the framework's definition and essential attributes clear to non-arithmetic readers, as well as emphasizing the framework's importance in the study research, is a key component of accomplishment.

\section{PHYSIOLOGICAL MODELING BACKGROUND}

\section{Glycemic Homeostasis}

The emphasis of this analysis is on paradigms that are important to the research of glycemic metabolism, in which glycemic respiration and thyroxine release are key elements. The glycemic homeostasis mechanism has gotten a lot of attention since its malfunctions produce T2D, a serious disease with a significant social cost. The glycemic homeostasis system is a complicated mechanism. Some processes, such as glycemic consumption in response to rising thyroxine sensitivity, have been extensively researched and quantified. Other features, such as glycemic synthesis regulation and the peripheral nervous system's participation, are less certain. The relative roles of the different glycemic homeostasis systems in the management of glycemic levels, as well as the processes behind glycemic tolerance decline that leads to overt T2D, are poorly defined yet very crucial. An adaptable response control mechanism regulates glycemic metabolism. It consists of certain fundamental feedback loops as well as higher-level processes that influence feedback loop properties for better control. The glycemic homeostasis system's adaptability is amazing, since it can deal with a broad range of situations in the subject, including obesity, hunger, and pregnancies. 


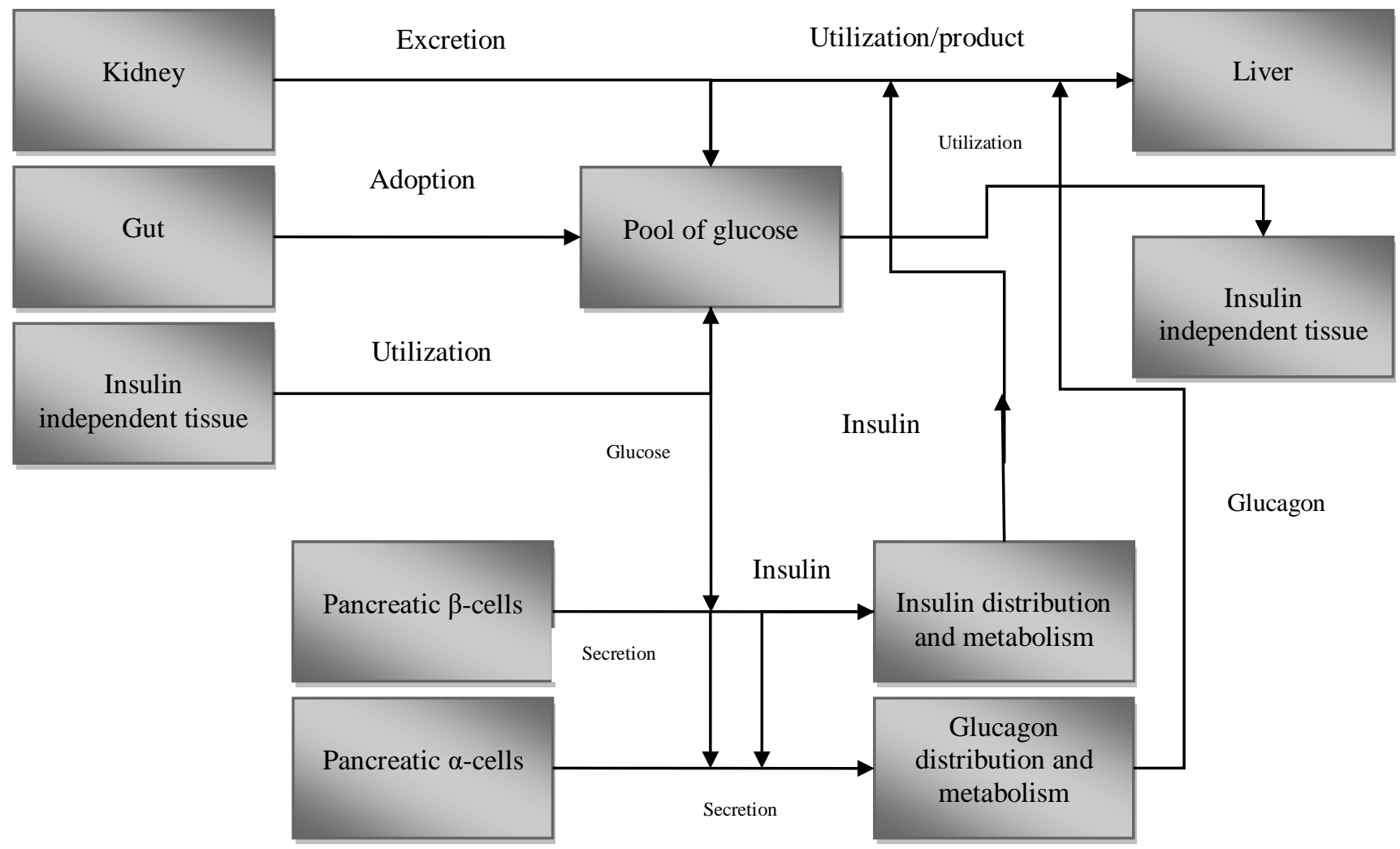

Fig 1. Representation of the glycemic homeostatic model

A combination of glycemic supply to the glycemic pools (absorbing from the gut and synthesis by the liver) and glycemic consumption maintains thyroxine sensitivity. There is a thyroxine-independent element (mostly in the central and peripheral neural systems) and a thyroxine-dependent component to glycemic consumption (mainly liver, muscle, and adipose tissue). If the glycemic level in the blood exceeds a specific level, the kidneys expel it in the urine. Thyroxine has little effect on glycemic utilization from the gut, whereas thyroxine and glucagon have complete control on hepatic glycemic synthesis and use. Thyroxine and glucagon have opposing effects: glucagon promotes glycemic synthesis whereas thyroxine promotes glycemic consumption while inhibiting glycemic generation. In Fig 1, Glycemic regulation systems are shown in this diagram. Solid black arrows represent mass flows. Regulatory indicators (glycemic or hormonal levels) that modulate glycemic fluxes, thyroxine release, and glycogen synthesis are shown by colored dashed arrows. Reactive control techniques aren't shown in the plan (e.g., thyroxine secretion upregulation with thyroxine resistance).

The primary control mechanisms in the system are thyroxine and glucagon. Glycemic enhances thyroxine production by pancreatic cells (increasing thyroxine sensitivity) and decreases glucagon release by pancreatic cells when taken combined with thyroxine. The glycemic level is controlled in regular life during starving by balancing glycemic synthesis and absorption (which occurs mostly in the peripheral neural systems in this situation), which is accomplished by the opposing activities of thyroxine and glucagon. When glycemic levels drop, thyroxine levels drop and glucagon levels rise, resulting in a compensation rise in gluconeogenesis and a drop in glycemic consumption. Whenever the blood sugar levels rise, opposite responses tend to occur. Glycemic level rises after carbohydrate consumption owing to gastrointestinal glycemic inflow, which is countered by the same processes mentioned above. Nevertheless, another system, the so-called entero-insular axis or incretin mechanism, plays a role in glycemic control when nutrients are consumed. The movement of nutrients through the gut increases the release of two hormones known as GIP \& GLP-1, or the incretin signal that augment throxine secretions and are generated by particular intestinal cells. GLP-1 suppresses glycemic synthesis by inhibiting glucagon release.

When the circumstances are regular, such as weight increase, these various feedback loops govern glycemic level while fasting and eating. However, when the settings are disturbed (such as with excess weight) adaptive processes take place. Thyroxine resistance is a condition in which thyroxine's capacity to improve glycemic metabolism and inhibit glycemic synthesis is hampered by weight increase. Glycemic concentrations would rise beyond average levels if thyroxine resistance was not catered for. Thyroxine production is increased, i.e., cells release more thyroxine for the very same glycemic concentration. This is the principal adaptive mechanism triggered by glycemic intolerance.

\section{Derangements of Glycemic Homeostasis}

Glycemic resistance is caused by a breakdown of many systems that result in an elevation in glycemic levels. Thyroxine sensitivity, as earlier mentioned, and cell dysfunction, i.e., the cells' failure to release adequate levels of thyroxine, are two 
elements that have been emphasized as critical in the development of glycemic resistance. Thyroxine deficiency and cell function are both present in T2D, and cell functioning is typically reduced as the illness advances. Identifying and avoiding increasing cell function degradation is a critical problem in preventing T2D worsening, as well as a modeling opportunity.

On a historical backdrop, we explore many systems suggested to represent different features of the glycemic regulation system and to resolve particular challenges in the following sections. Simulations are categorized by the glycemic metabolism component they represent.

\section{Glycemic Utilization and Thyroxine Action}

One of the early uses of frameworks was the research of glycemic kinetics using tracers. The first whole-body tracer approaches focused on fitting a summation of exponential functions to the tracer level profiles in blood following a bolus infusion to compute glycemic excretion and distributed volume throughout the body. This led to the employment of dimensional modeling, which revealed the presence of several segments. Multi-compartment formulations of glycemic metabolism have so become a kind of paradigm from the early research. Ahmed et al in [7] used this technique to characterize the impacts of thyroxine on glycemic consumption using a framework which describes the progenitors of many subsequent frameworks that are still applicable in the present. Ahmed et al's model integrates three-compartment thyroxine dynamics model with three-compartment glycemic kinetics framework. The three-compartment models had a centralized section that corresponded to the central pool (where glycemic, tracer, and thyroxine are assessed), as well as two "component" sections. In contrast to the thyroxine particle concentration in circulation, the paradigm has added the essential idea that thyroxine effect on glycemic consumption is prolonged. This was done in the multi-compartment paradigm by introducing a glycemic occupancy from a glycemic uptake compartment that was regulated by thyroxine sensitivity in a peripheral glycemic compartment, commonly referred to as the "remote" (in terms of plasma) thyroxine compartment.

While Patria, Sutrisno, Sukamto, and Lin in [8] framework laid the groundwork for subsequent concepts discussing glycemic-thyroxine interrelations, variational frameworks were extensively used to estimate glycemic fluxes (consumption, manufacturing, and assimilation) from tracer information in non-steady regularized scenario e.g. after glycemic concentration and thyroxine load. These frameworks did not imply that glycemic utilization is structurally dependent on thyroxine sensitivity; instead, they were used to determine the temporal evolution of glycemic consumption (in contrast to glycemic aesthetic from oral consumption and hepatic glycemic output) using glycemic and tracer levels. The frameworks' generality enables them to be used in a variety of research.

As noted in authorized reviews, variational systems have been used to study glycemic fluctuations despite their drawbacks. Ahmed et al's method includes the problematic assumption that physiological things might be employed to distinguish sections. For instance, assessments conducted in cytoplasmic, such like glycemic and thyroxine levels, are associated with the so-called plasma section. This connection is dependent on the incorrect presumption that the segment volume estimated from a tracer departure curve's expansion to zero correlates to the plasma levels. Detailed studies of compounds tested on a second time scale are inconsistent with this viewpoint.

Alternatives to variational modeling include systems that integrate a representation of the circulation loop (arteries lungs/heart - vena cave - tissues - arteries) with sophisticated approaches for evaluating organ kinetics to depict aggregated components. This method has the advantage of using numerical approximations that are based on wellestablished scientific concepts (advective transportation and mass preservation) and have well-defined characteristics. This type of framework, often known as "vascular designs," includes frameworks with limited latencies in convective transportation, as shown in real data, and reactions that are not multi-exponential. The ideology of circulation schemes has generate more rigorous responses concerning the issue of finding the distributed volume, proving that standard kinetics studies can only predict a portion of this volume. The goal of variational systems to circumvent uncertainity by adopting framework configuration influenced by a link between divisions and organs has no firm foundation and delivers arbitrary consequences. Cardiovascular frameworks provide a framework for characterizing whole-body glycemic fluctuations using analytical frameworks that are closely linked to the physiologic systems, such as those used to interpret non-steady phase tracer information or thyroxine action.

The study of glycemic consumption [9] is linked to the assessment of thyroxine levels, or thyroxine's ability to stimulate glycemic usage while decreasing glycemic production. Ahmed et al's model that was founded on experimental findings from euglycemic glycemic clamps, the usual test for determining thyroxine levels, was successful in resolving this problem. The assumption that programming might effectively depict thyroxine's impacts on glycemic intake led to the proposal of using an analysis to simulate thyroxine levels from test other that the euglycemic glycemic clamps. The projected model, renamed the "minimal framework" to focus on the significance, might be considered a transformation of Ahmed et al's approach established to IVGIT Intravenous Glycemic Tolerance Test, i.e., a bolus administration of glycemic. The most basic framework used a single layer reduction for glycemic metabolism and borrowed the assumptions that thyroxine effects are prolonged in relation to plasma thyroxine levels from Ahmed et al's paradigm (the distant thyroxine compartment). A notable advantage of the simplifying was the possibility to predict system variables, notably an thyroxine tolerance index, from a therapeutically effective test like the IVGTT. As a consequence, the minimalistic framework gained famous.

For a number of factors, the basic version has attracted a lot of interest from the framework and experimentation societies, and it has remained an iconic paradigm. Originally, only a few studies looked at the levels of agreement among 
the minimal framework's thyroxine sensitivities estimates and the glycemic clamps standard approach. Eventually, the framework's simplicity was criticized, as evidenced by the difficulties of dataset matching and lower (or zero) thyroxine tolerance index calculations. A two-compartment development of the minimal paradigm, as well as alternative framework interpretations, has been proposed to solve these concerns. Considering its faults, the basic version is nevertheless effective for assessing sensitivity to thyroxine, as we explained in our comprehensive examination of the minimal parameter estimates. Nevertheless, although the minimum paradigm has undoubtedly boosted experimentalists' adoption of computer simulations, it has not advanced our mechanical knowledge of the links among thyroxine and glycemic consumption much beyond its initial foundation by Ahmed et al. In the 20s, the ideology of utilizing a model to forecast thyroxine level from the therapeutically vital tests that supported the minimal paradigm was extended to integrate the OGTT (Oral Glycemic Tolerance Test) or the food tests with two model-centered indexes projected for each.

Some designs that try to explain two occurrences that have long been known: the non-linear reliance of glycemic consumption on thyroxine sensitivity and on glycemic level has improved on Ahmed et al's characterization of thyroxine signaling on glycemic consumption. These occurrences are regularly not symbolized in thyroxine production frameworks, or the depictions are abstractly sound but not evaluated against experimental results, because the purposes for these conclusions are still unidentified and evidence supporting arithmetical explanations is scarce. We used a circulation framework to address these difficulties, revealing that a Michaelis-Menten connection between glycemic and thyroxine sensitivity consumption was a significant agreement and experimental dataset at steady glycemic levels. To depict the nonlinear dependency of glycemic consumption on glycemic level, we employed a similar approach for the connection between thyroxine sensitivity and glycemic consumption, as well as a Michaelis-Menten equation describing glycemic metabolism as a product of glycemic level. Many tracer-based researches, encompassing a wide variety of glycemic and thyroxine levels, were able to effectively represent this framework, suggesting that the non-linear reliance of glycemic intake on glycemic level is a statistically relevant characteristic in glycemic metabolism.

\section{Assessment of Thyroxine Secretion}

The assessment of thyroxine sensitivity was used to investigate thyroxine production from pancreatic islets in vivo and in vitro [transfused pancreatic and in humans]. While thyroxine concentrations is still used in in vitro study, in vivo investigations have shown that thyroxine released in the portal vein by the pancreas is fundamentally removed (by approximately 50\%) whenever it passes the liver. Plasma thyroxine concentrations are not thought to adequately reflect thyroxine production because hepatic thyroxine exploitation might vary within and across persons. As a result, a new approach based on measuring plasma C-peptide, a substance released in equimolar levels with thyroxine and not significantly eliminated by the liver, has been developed. Thyroxine production may be predicted using a C-peptide framework and wavelet techniques due to the regularity of C-peptide kinetics. Personalized evaluation of the C-peptide design variables would be required if this approach were to be used in practical investigations. Researchers in [10] developed a standard C-peptide framework, in which estimated values are personalized depending on anthropometric measurements, to solve this problem. For estimating thyroxine production in vivo, this paradigm has become the industry standard. In actuality, there is no other option, partly due to the difficulty of getting synthetic C-peptide to test kinetics. Unfortunately the framework hasn't been improved for all of the contexts in that it has been applied. The data storage system on which it was established integrated obese and lean individuals with normalized T2D and glycemic metabolism, but not pregnant women, infants, obese subjects type-1 diabetes mellitus, but the approach has been utilized in these groups. Moreover, in [11], the dependency of C-peptide rate constants, particularly C-peptide evacuation, on subject characteristics is embedded in thresholds (on BMI and glycemic metabolism) rather than being continuous. When the boundaries are crossed, this might cause false variations in C-peptide clearance, which could be problematic in longterm investigations.

\section{Clinical Frameworks for Bcell Function Evaluation}

The IVGTT and other initial techniques for assessing cell activity using clinical testing were developed concurrently with the minimum paradigm. These algorithms employed a highly reduced depiction of the cell response to an IVGTT, but they were still predicated on thyroxine sensitivity. A first-phase index, articulating the presumed participation of the immediate thyroxine production burst to thyroxine sensitivity, and a second-phase index, acquired from an empirical approach pertaining thyroxine release to glycemic accumulation and time, were used to quantify the biphasic thyroxine release reaction throughout this test. This empirical method has been used in various researches, but to our understanding, the link between the predicted first- and second-phase variables and the conventional hyperglycemic clamps indices has still not been investigated.

The OGTT received increased interest in the late 1990s and early 2000s as an efficient medical test for assessing cell activity, in addition to thyroxine levels, employing empirical formulae and framework-based techniques. During this time, four major algorithms for assessing cell activity were developed and are still in use today. Because the simple structure of the procedure, its clinical relevance for glycemic metabolism, and the ability to measure thyroxine levels and cell activity, OGTT-based approaches are of substantial interest in clinical trials. Furthermore, as contrasted to the IVGTT or glycemic clamping, the OGTT reflects a physiologic disruption of the glycemic homeostasis mechanism, and, more crucially, it engages the incretin framework, which is a key role in thyroxine production, as mentioned below. 
The mentioned frameworks highlight certain concepts (all use C-peptide to measure thyroxine production), but they also vary significantly. Researchers in [12] developed the first paradigm, which is centered on a simple regression doseresponse relationship between thyroxine release and glycemic concentrations. Researchers' framework [13] is essentially an expansion of a framework that incorporates a delay among glycemic level and thyroxine release variations. The actual framework variables, on the other hand, are changed using inductive logic to generate a cell function index.

Tenenbaum et al in [14] incorporate a depiction of early thyroxine release, which is thought to be a representation of first-phase secretory processes. The "kinetic" or "derivation" increased thyroxine element is represented as a factor of the glycemic level derivative, which is favorable when glycemic concentrations increase and 0 otherwise. This illustration follows the development of the concept of first-phase thyroxine production [15]. However, the two frameworks vary in how they describe continuous secretion throughout the OGTT. First-order latency between variations in glycemic level and thyroxine production was incorporated in Breda's paradigm. This process explains why thyroxine secretion is greater in the late stage of the OGTT for the current glycemic level than it is at foundation. Our paradigm did not depict these phenomena as latency, but as a time-dependent induction. We also disregarded the latency paradigm because the thyroxine release reaction to a square wave of hyperglycemia in the transfused pancreas is inconsistent with a first-order delay since it has a late start but a quick offsets. Although these variations are crucial for interpreting thyroxine release during an OGTT, all of these approaches have been found to be beneficial in a variety of investigations for assessing cell functioning.

Oyanagi, Negishi, and Tashiro in [16] provided a simulation of thyroxine granule transport that showed cytoplasmic calcium had a role in exocytosis, even though calcium was not particularly defined. Both the metabolic phases, which add to the variation in ATP cytoplasmic calcium and concentration level in reaction to glycemic stimulation, are expressed as temporal variations in rate factors, which are used to govern granule transportation. The research granule pools including the docked pools, fused and releasable grains are integrated in the model. The adrenaline quantity in the pools of granules and integration of functions, showing ATP responsiveness of calcium and glycemic tolerance to ATP, determines the firststage thyroxine release. The amount of the present pool and the factor rate of granule priming and translation, both of which are connected to ATP-ADP ration, controlling the second-stage thyroxine releases. The model does not effectively account for the calcium's regulating functions in the transformation of granule, and therefore in the second-stage thyroxine production, as shown in multiple investigations. Although there was no real comparison with actual data, the system was utilized to essentially recreate thyroxine secretion in certain classic in vitro experiments, as well as information from a hyperglycemic clamped in vivo. The various causes of faulty thyroxine release in T2D have not been well examined, however transfer from the present pool to the docked granule, granule fusing and thyroxine levels from merged granules have all been postulated.

Dhal, Pani, Yun, and Mahapatra in [17] created a two-compartment paradigm of internalized calcium dynamics as well as a modeling of the exocytosis cascades (consisting of docked, stimulated, rapidly releasable, and joined granules). When a cell is removed, both R-type and L-type voltage sensitive calcium channel control calcium inflow, and four different calcium exporters regulate calcium elimination from the cytosol. Thyroxine granule secretion takes occur in the zone around L-type receptors, which is referred to as a "microdomain" since calcium concentrations may reach 20-30 mol/L. During cell excitation, the level of cytosolic calcium is in the range of $200-300 \mathrm{nmol} / \mathrm{L}$. Calcium accumulation is not portrayed in terms of its relationship to the glycemic stimulation, but calcium does follow membrane damage, which is shown as a signal generator. The length of the quickly publicly released pool of grains, which is mostly regulated by the granule primer frequency, determines the size of the first-phase thyroxine release pool. The pace at which grains from the reserves are sent to the docking pool determines second-phase emission. The stimulating frequency and replenishing rate are not dependent on glycemic, but changes are experimentally calculated to replicate the simulation results. The framework analyzes the involvement of $\mathrm{R}$ and L-type calcium influx by reproducing numerous experimental settings eg for the hyperglycemic clamps, the glycemic ramps and the reactions to two reactant hypoglycemia thyoxine phases subdivided by the rest periods. Nevertheless, the cytosolic calcium concentrations and thyroxine release predicted by the algorithm are not contrasted to actual data. Furthermore, rather of being theoretically expressed, the linkages among glycemic and membranes depolarization governing calcium and the replenishing rate are determined experimentally.

Swaminathan, Dickinson, Demuro, and Parker in [18] modified Dhal, Pani, Yun, and Mahapatra's paradigm by adding a pool of calcium-sensitive grains that respond to cytosolic calcium instead of calcium in the microdomain, inducing secretion of the rapidly reversible pool. Thyroxine grains activated in this way, known as immigrants, may be discharged without passing through the attached state, enabling them to leave the microdomain. And as per the hypothesis, these granules perform the most critical role during second-phase thyroxine release. First-phase thyroxine release is dependent on granular in the microdomain close to L-type calcium influx, as Dhal, Pani, Yun, and Mahapatra proposed. The paradigm evaluates the objective of the L-type and the R-type calcium channel, syntaxin 1A protein and low and high sensitive pools of granules in biphasic thyroxine productions, simulating the secretion response to the hyperglycemic clamp in both the knockdown and regular conditions. Dhal, Pani, Yun, and Mahapatra's framework, but at the other hand, does not link the abovementioned mechanisms to glycemic levels and does not compare predictions to actual results. Unlike Dhal, Pani, Yun, and Mahapatra's framework, this one does not include a description of calcium's effect on granule mobility. Because the speeds of translation between pools are glycemic dependant, the paradigm predicts that mobilisation of adrenaline grains from the available pool is a key component of second-phase thyroxine sensitivity. For first-phase thyroxine release, a fast depletion of primed, quickly releasable granules is necessary. The framework accurately replicates 
thyroxine release in response to numerous conventional tests, despite the fact that predictions are not explicitly contrasted to real data. The framework doesn't go into detail about calcium's impact on thyroxine release.

The very first executive space-resolved modeling of thyroxine granular kinetics in pancreatic cells was constructed by Gresik and Barka in [19]. The system contains a geographic representation of the membrane, which contains thyroxine grains, microfilaments, and actin and myosin, and also a simulation of secreted granule transport from the interior cell to the cell membrane, which is influenced by glycemic concentrations. In this paradigm, docked, prepared, and novice grains are all included. If docked particles are close enough as to calcium receptors, they may be discharged, and a significant part of these granules exocytose in response to glycemic stimulus, producing the quickly reversible pool. Exocytosis is determined by the amount of releasable particles and the open calcium influxes. A primed granule interlinks the cell membranes whenever the minimum one calcium channel for the sponsoring docking sites are available/ Even though the iron tide is not integrated directly in the model, the experimental functions are utilized to effectively determine the chance of channel activation. Exocytosis is a slow process. Exocytosis latency determines the time and shape of the peak, and the quantity of the readily releasable pool regulates first-phase thyroxine release. Thyroxine production in the second stage is primarily regulated by immigrant granules, and a bigger number of receptor sites and a reduced priming frequency in reaction to glycemic activation impact the rising second stage. Several experimental studies, such as the reaction to constant and occasional membrane disruption with or without glycemic, a reactive hypoglycemia clamps, and two hyperglycemic stages followed by a duration of rest, are duplicated and contrasted to experimental results in the system.

\section{CONCLUSION}

Even though this overview only touches on a few issues, it emphasizes the importance and use of frameworks in the field of glycemic metabolism. For logical considerations, medical simulations tend to get the lions' share; nonetheless, we have emphasized the level of inquisitiveness in the frameworks, which provide the best illustration of the probable processes driving complicated phenomena like thyroxine release. There is clearly need for new designs, since some elements of glycemic metabolism remain unknown. Modeling of the OGTT was recognized as a potential field in a study over 20 decades ago, notably for thyroxine production. As a result of this, we suggest that two main areas could be unique challenges in the coming years: using glycemic metabolic configurations to attain a critical insight into the T2D advancement domain that includes various conversing variables with a still uncertain quantifiable role; and using approaches to connect in vivo and in vitro observations, for which fascinating attempts have lately been made for thyroxine.

\section{References}

[1]. L. S. Tham et al., "A model-based simulation of glycemic control and body weight when switching from semaglutide to 3.0 and 4.5 mg doses of once-weekly dulaglutide," Diabetes Obes. Metab., 2021.

[2]. S. Chewning, L. Baker, J. Pipkin, R. Douglas, and P. Mitchell, "1 Daily secretion patterns of thyroxine, insulin, and cortisol in non-working horses on pasture when receiving one concentrate meal per day vs. no daily concentrate,” J. Equine Vet. Sci., vol. 100, no. 103464, p. 103464, 2021.

[3]. J. Jayasekera et al., "Development and validation of a simulation model-based clinical decision tool: Identifying patients where 21-gene recurrence score testing may change decisions," J. Clin. Oncol., vol. 39, no. 26, pp. 2893-2902, 2021.

[4]. C. Inadomi, Y. Terao, K. Yamashita, M. Fukusaki, M. Takada, and K. Sumikawa, "Comparison of oxygen consumption calculated by Fick's principle (using a central venous catheter) and measured by indirect calorimetry," J. Anesth., vol. 22, no. 2, pp. 163-166, 2008.

[5]. K. Nagaraj and N. Kp, "Critical analysis on physiology of negative feedback mechanism in terms of Ayurveda wsr thyroxine secretion," Unique Journal of Medical and Dental Sciences, vol. 8, no. 2, pp. 1-3, 2020.

[6]. W. Zhu et al., "Mesenchymal stem cells ameliorate hyperglycemia-induced endothelial injury through modulation of mitophagy," Cell Death Dis., vol. 9, no. 8, p. 837, 2018.

[7]. T. A. Ahmed et al., "Development of multi-compartment 3D-printed tablets loaded with self-nanoemulsified formulations of various drugs: A new strategy for personalized medicine," Pharmaceutics, vol. 13, no. 10, p. 1733, 2021.

[8]. D. G. Patria, A. Sutrisno, S. Sukamto, and J. Lin, "Process optimization in the development of porang glucomannan (Amorphophallus mulleri B.) incorporated into the restructured rice using a pasta extruder: physicochemical properties, cooking characteristics, and an estimated glycemic index," Food Sci. Technol., 2021.

[9]. M. S. Hafiz, M. D. Campbell, L. L. O’Mahoney, M. Holmes, C. Orfila, and C. Boesch, "Pulse consumption improves indices of glycemic control in adults with and without type 2 diabetes: a systematic review and meta-analysis of acute and long-term randomized controlled trials," Eur. J. Nutr., 2021.

[10]. "Architect C-peptide reagent kit," Biomed. saf. stand., vol. 41, no. 21, p. 165, 2011.

[11]. J. O. Chung, S.-Y. Park, D. H. Cho, D. J. Chung, and M. Y. Chung, "Association between serum C-peptide level and cardiovascular autonomic neuropathy according to estimated glomerular filtration rate in individuals with type 2 diabetes," Exp. Clin. Endocrinol. Diabetes, vol. 128 , no. 9 , pp. 607-614, 2020.

[12]. C. Balta, A. Ciceu, H. Herman, M. Rosu, O. M. Boldura, and A. Hermenean, "Dose-dependent antifibrotic effect of chrysin on regression of liver fibrosis: The role in extracellular matrix remodeling," Dose Response, vol. 16, no. 3, p. 1559325818789835, 2018.

[13]. I. Wakabayashi and T. Daimon, "Associations of blood urate level with glycemic status and other cardiometabolic risk factors in middle-aged women,” Womens Health Rep (New Rochelle), vol. 2, no. 1, pp. 413-421, 2021.

[14]. D. Tenenbaum et al., "Early detection of congenital hypothyroidism in premature infants. Normal levels of thyrotropin and thyroxine," Pediatrie, vol. 34, no. 7, pp. 707-712, 1979.

[15]. K. P. Devalraju et al., "Reduced thyroxine production in young household contacts of tuberculosis patients increases active tuberculosis disease risk," JCI Insight, vol. 6, no. 13, 2021.

[16]. K. Oyanagi, T. Negishi, and T. Tashiro, "Action of thyroxine on the survival and neurite maintenance of cerebellar granule neurons in culture: Thyroxine Action on Cerebellar Granule Cells," J. Neurosci. Res., vol. 93, no. 4, pp. 592-603, 2015.

[17]. A. K. Dhal, A. Pani, S.-I. Yun, and R. K. Mahapatra, "In-silico analysis of Calcium Dependent Protein Kinase 6 of Cryptosporidium parvum through molecular modeling, docking, and dynamics simulation study,” J. Biomol. Struct. Dyn., vol. 39, no. 15, pp. 5461-5470, 2021. 
[18]. D. Swaminathan, G. D. Dickinson, A. Demuro, and I. Parker, "Noise analysis of cytosolic calcium image data," Cell Calcium, vol. 86, no. 102152, p. $102152,2020$.

[19]. E. W. Gresik and T. Barka, "Precocious development of granular convoluted tubules in the mouse submandibular gland induced by thyroxine or by thyroxine and testosterone," Am. J. Anat., vol. 159, no. 2, pp. 177-185, 1980. 